\title{
Massive star formation in the outer Galaxy: $\mathrm{S} 284$
}

\author{
E. Puga ${ }^{1}$, C. Neiner ${ }^{2}$, S. Hony ${ }^{3}$, A. Lenorzer ${ }^{4}$, A.-M. Hubert ${ }^{2}$ \\ and L. B. F. M. Waters ${ }^{5}$ \\ ${ }^{1}$ Katholieke Universiteit Leuven, Celestijnenlaan 200D, 3001 Leuven, Belgium, \\ email: elena@ster.kuleuven.be \\ ${ }^{2}$ GEPI, UMR 8111 du CNRS, 5 place Jules Janssen, 92195 Meudon Cedex, France, \\ ${ }^{3}$ CEA Saclay, Bat.609 Orme des Merisiers, 91191 Gif-sur-Yvette, France, \\ ${ }^{4}$ Instituto Astrofysico de Canarias,C/ Vía Láctea s/n, E-38200, La Laguna, Spain, \\ ${ }^{5}$ Universiteit van Amsterdam, Kruislaan 403, 1098SJ Amsterdam, The Netherlands
}

\begin{abstract}
S284 is a diffuse HII region located in the anti-centre of the galactic disk $(l=212$, $\mathrm{b}=-1.3$ ), at a distance of $5.5 \mathrm{kpc}$ and it is relatively isolated. S284 harbours a cluster of stars in its centre known as Dolidze 25 (also known as C 0642+0.03). A spectroscopic study of the cluster sources (Lennon et al. 1990, A\&A, 240, 349) revealed: a) the spectral types of these central sources and b) the low metallicity of the cluster (a factor six lower than the solar metallicity). The age of the cluster $(6 \mathrm{Myr})$ has been determined through isochrone fitting of precision photometry (Turbide \& Moffat, 1993, AJ, 105, 1831).

We have conducted Spitzer-IRAC observations of $0.9 \times 1.2$ degrees around S284 in the four IRAC bands $(3.6,4.5,5.8$ and $8.0 \mu \mathrm{m})$. These data provide us with an unprecedented perspective of the dust component toward S284. We also present complementary observations with the WFC-INT (Roque de los Muchachos) in the $\mathrm{H} \alpha$ filter. The inspection of the images reveals the existence of: i) Rings of dust that constraint the ionised gas, ii) Elephant trunks stretching toward the centre of the main HII region, namely the cluster Dolidze 25 and iii) various spatial scales (4 main shells and 22 knots).

We have calculated the dynamical age of the central HII region (S284) in a very simple pressuredriven scenario, using the strong-shock approximation. The resultant age of the main shell is 8 Myr, in good agreement with the estimated age of the central cluster (Dolidze 25). The presence of another two smaller ionised bubbles (with diameters of 3' and 5', respectively) located at the rim of this HII region suggests a later generation of high-mass star-forming regions. The color-color diagram of the IRAC photometry betrays the existence of several Class I objects, most of which are located at the rim of the most prominent shell.

Multi-wavelength photometry has been gathered to construct the Spectral Energy Distribution of the 22 blobs detected toward S284. The SEDs reveal luminosities corresponding to low- and intermediate-mass stars.

We conclude that the remarkable symmetry of the main HII bubble, the spectra of masses harboured by the different blobs around it, and most importantly, the uniform presence of elephant trunks that stretch toward the centre can be best explained by the growth of dynamical instabilities in a collected layer (Garcia-Segura \& Franco, 1996, ApJ, 469, 171). In the context of this hypothesis, the low metallicity of the environment would significantly determine the length and stability over time of the elephant trunks.
\end{abstract}

Keywords. stars: formation, (ISM:) HII regions 